\title{
Le plan canadien de gestion de la rage : une approche intégrée de coordination des activités de lutte contre la rage au Canada
}

\author{
Tataryn $\mathrm{J}^{1 *}$, Buck PA ${ }^{1}$
}

\section{Résumé}

Bien que les cas de rage chez les humains soient exceptionnellement rares au Canada, la rage demeure endémique chez certaines populations animales, nécessitant ainsi une vigilance continue. La rage a toujours été une responsabilité partagée par les autorités locales, provinciales et territoriales, et fédérales, comme en témoigne le Plan canadien de gestion de la rage de 2009. Depuis 2009, un certain nombre de changements ont été apportés dans la gestion de la maladie, y compris la mise au point de nouveaux tests, d'un vaccin antirabique à prise orale pour la faune, des leçons apprises de cas récemment observés chez les animaux et le transfert des responsabilités des administrations fédérales-provinciales-territoriales en 2014. Les ministères et les organismes fédéraux continuent de soutenir la gestion de la rage au moyen de diverses activités. Comme la situation de la rage continue d'évoluer, les stratégies et les cadres requis pour la gestion de cette maladie doivent suivre le pas, ce qui explique la présente révision du Plan canadien de gestion de la rage au Canada et du Plan nord-américain de gestion de la rage.

\author{
Affiliation \\ 1 Centre des maladies \\ infectieuses d'origine alimentaire, \\ environnementale et zoonotique, \\ Agence de la santé publique du \\ Canada
}

*Correspondance : joanne. tataryn@phac-aspc.gc.ca

Citation proposée : Tataryn J, Buck PA. Le plan canadien de gestion de la rage : une approche intégrée de coordination des activités de lutte contre la rage au Canada. Relevé des maladies transmissibles au Canada 2016;42:151-2. https://doi.org/10.14745/ccdr.v42i06a05f

\section{Introduction}

La rage demeure une menace redoutable en matière de santé publique dans de nombreuses régions du monde en développement. Malgré la disponibilité d'outils et d'approches stratégiques visant à éliminer les cas de rage humaine transmise par les chiens, la maladie tue des dizaines de milliers de personnes chaque année (1). En revanche, les cas humains ont été exceptionnellement rares au Canada au cours des dernières décennies en raison de la réussite des efforts collectifs déployés par les divers partenaires du domaine de la santé des humains, de la faune et des animaux domestiques.

Malgré ces succès, la rage continue de représenter une menace pour la santé publique et les populations animales au Canada, et notre travail de lutte contre la rage est loin d'être terminé. Il existe encore des régions au Canada où la rage chez la chauve-souris est endémique et certaines régions connaissent de nouvelles incursions de rage chez des animaux terrestres. De nouveaux défis anthropiques, comme le déplacement d'animaux et les effets de la mondialisation, se sont ajoutés à la complexité séculaire de la dynamique de la transmission de la rage dans les écosystèmes. Le déplacement d'animaux atteints de la rage peut rapidement causer des incidents à l'échelle nationale et internationale nécessitant une communication et une coordination rapides entre un grand nombre d'intervenants pour une gestion efficace du problème et la protection du public.
Les autres défis importants liés à la prévention et à la maîtrise de la rage au Canada sont le manque d'accès à des services vétérinaires dans le nord du pays, les effets des changements climatiques, et l'étendue et les interactions variables des populations selvatiques (faune). Des efforts collectifs et coordonnés des représentants dédiés des secteurs de la santé des humains, de la faune et des animaux domestiques seront nécessaires pour surmonter ces défis.

\section{Responsabilité partagée}

La rage a toujours été une responsabilité partagée par les autorités locales, provinciales et territoriales, et fédérales. En 2009, des organismes de la santé publique et de l'agriculture, des organismes provinciaux et territoriaux de la faune, l'Agence de santé publique du Canada (ASPC), I'Agence canadienne d'inspection des aliments ( $\mathrm{ACIA})$, Environnement Canada ainsi que des organismes clés non gouvernementaux ont élaboré conjointement le Plan canadien de gestion de la rage, lequel dresse une stratégie nationale pour la gestion de la rage. $\mathrm{Ce}$ plan décrit les stratégies de surveillance, de prévention et de maîtrise des variantes du virus de la rage terrestre et associée à la chauve-souris au Canada, ainsi que les interventions requises.

En 2014, les rôles et les responsabilités des administrations fédérales-provinciales-territoriales ont changé, particulièrement quant à la collecte et à la soumission des échantillons, ainsi qu'aux enquêtes et à la mise en quarantaine des animaux 
domestiques soupçonnés d'être infectés (2). En outre, pour assumer ses responsabilités, les autorités provinciales et territoriales continueront d'être responsables de la gestion de la rage et de la santé humaine. Les divers ministères et organismes fédéraux continuent de soutenir la gestion de la rage au moyen d'activités clés comme le dépistage, l'approbation et l'approvisionnement des vaccins, les tests sérologiques chez les humains, la prodigation de conseils sur la vaccination et le traitement des humains, la diffusion de messages de prévention clés et le signalement des cas à l'échelle nationale. D'autres partenaires demeurent une partie intégrante de la prévention et de la gestion de la rage, y compris les organismes non gouvernementaux de protection de la faune, les vétérinaires privés, les communautés touchées par la rage et le grand public.

\section{Nouveaux développements}

De nouveaux tests et de nouvelles technologies pour la maîtrise de la rage ont été mis au point, notamment un vaccin oral antirabique efficace contre les mouffettes et les ratons laveurs, et diverses leçons ont été tirées des récentes éclosions. En conséquence, un groupe de travail composé d'autorités fédérales, provinciales et territoriales de la santé publique, de l'agriculture et de la faune, et d'intervenants d'organismes non gouvernementaux, a été délégué pour mettre à jour le Plan canadien de gestion de la rage. Collectivement, les membres de ce groupe représentent un large éventail de disciplines, y compris l'élaboration de politiques et de réglementation, l'épidémiologie, l'écologie, la surveillance et de la maîtrise des maladies infectieuses et les diagnostics en laboratoire. Dirigé conjointement par des représentants de l'ASPC et du ministère de la Santé et des Soins de longue durée de I'Ontario, ce processus aura pour objectif de s'assurer que le plan reflète les progrès et les changements au Canada et comprend de nouvelles idées et de nouveaux détails sur la coordination multijuridictionnelle des incidents se produisant à l'échelle nationale. La date d'achèvement cible de cette mise à jour du plan est l'automne 2016.

Le Plan canadien de gestion de la rage mis à jour reflétera les pratiques actuelles et fournira également un important contexte canadien pour la révision du Plan nord-américain de gestion de la rage (PNAGR) [3], qui est également en cours. Le PNAGR de 2008 a établi un protocole pour la gestion de la rage en Amérique du Nord et a joué un rôle clé dans la planification, les communications et les interventions transfrontalières mutuelles, facilitant ces activités au cours des dernières années. Il a également soutenu la prévention et la maîtrise de la rage dans chacun des pays membres ainsi que dans l'ensemble de l'Amérique du Nord.

\section{Conclusion}

La gestion efficace de la rage au Canada et en Amérique du Nord nécessite une approche intégrée et concertée entre les différents partenaires responsables de la santé humaine et animale. Comme la situation de la rage continue d'évoluer, les stratégies et les cadres requis pour la gestion de cette maladie doivent suivre le pas. Le Plan canadien de gestion de la rage, ainsi que les efforts de collaboration associés, sont positionnés pour relever ces défis toujours en évolution au cours des années à venir.

\section{Remerciements}

Les auteurs aimeraient remercier les nombreux partenaires et intervenants participant à la surveillance, à la prévention et à la maîtrise de la rage, ainsi qu'aux activités de sensibilisation, de recherche et d'intervention sur la maladie, partout au Canada.

\section{Conflit d'intérêts}

Aucun.

\section{Références}

1. World Health Organization. Rabies. World Health Organization. http://www.who.int/rabies/en/.

2. Canada Food Inspection Agency. Rabies Program Adjustments April 2014. http://www.inspection. gc.ca/animals/terrestrial-animals/diseases/reportable/ rabies/2014-03-31/eng/1396281140496/1396281190 676. (Disponible en français : http://www.inspection. gc.ca/animaux/animaux-terrestres/maladies/declarationobligatoire/rage/2014-03-31/fra/1396281140496/13962811 90676).

3. North American Rabies Management Plan: A Partnership for Effective Management. 2008 https://www.aphis.usda. gov/wildlife_damage/oral_rabies/downloads/Final\%20 NARMP\%209-30-2008\%20(ENGLISH).pdf. 